\title{
Squashed Embedding of E-R Schemas in Hypercubes
}

\author{
Chaitanya K. Baru* and Piyush Goel \\ Advanced Computer Architecture Laboratory, Department of Electrical Engineering and Computer Science, \\ The University of Michigan, Ann Arbor, Michigan 48109
}

\begin{abstract}
We have been investigating an approach to parallel database processing based on treating Entity-Relationship (E-R) schema graphs as dataflow graphs. A prerequisite is to find appropriate embeddings of the schema graphs into a processor graph, in this case a hypercube. This paper studies a class of adjacency preserving embeddings that map a node in the schema graph into a subcube (relaxed squashed or $R S$ embeddings) or into adjacent subcubes (relaxed extended squashed or RES embeddings) of a hypercube. The mapping algorithm is motivated by the technique used for state assignment in asynchronous sequential machines. In general, the dimension of the cube required for squashed embedding of a graph is called the weak cubical dimension or WCD of the graph. The RES embedding provides an RES-WCD of $O\left(\left\lceil\log _{2} n\right\rceil\right)$ for a completely connected graph, $K_{n}$, and RS embedding provides an RS-WCD of $O\left(\left\lceil\log _{2} n\right\rceil+\left\lceil\log _{2} m\right\rceil\right)$ for a completely connected bigraph, $K_{m, n}$. Typical E-R graphs are incompletely connected bigraphs. An algorithm for embedding incomplete bigraphs is presented. 1990 Academic Press, Inc.
\end{abstract}

\section{INTRODUCTION}

This paper presents one aspect of a novel approach to database processing which is based on treating schema graphs as dataflow graphs and then mapping such graphs onto a set of processors to support parallel database processing. Entity-Relationship, or E-R, schema graphs [10] and hypercube multicomputers are considered here. Adjacent nodes (object classes) in the schema graph are mapped to adjacent subcubes in the hypercube. This type of mapping is referred to as relaxed squashed embedding.

Semantic data models were introduced as aids to the easy modeling of data and the various interrelationships among them. The E-R model is a well-known example of such a data model. Many other semantic data models have also been proposed, including variants of the E-R model [15, 29] and other models such as SDM [19], SAM* [27], NIAM [30], and IFO [1]. Semantic data models typically support constructs such as aggregation, generalization, and classification. Most models are graph-oriented; i.e., the various interrelationships among data objects are represented

\footnotetext{
* Partially supported by Grant IRI-8710855 from the National Science Foundation.
}

via a graph employing several different symbols to identify different object and relationship types. This graph is referred to as the logical schema graph. Figure 1 shows a logical schema graph for a simple database using a variant of the E-R model, viz. the Entity-Category-Relationship (ECR) model [15].

An important concept in the work presented here is the use of the logical schema graph as the physical representational structure; i.e., the data are directly stored in the computer in the form indicated by the schema graph. Each node in the graph represents a collection of objects or relationships among objects. These are stored directly as files, either flat or structured. Several points should be noted here. First, user queries can always be represented as subgraphs of the schema graph, with appropriate selection predicates at each node. Second, a dataflow processing approach can be employed by treating the query graph as a dataflow graph. Third, the dataflow processing is made more efficient if each node of the graph resides on a different processor with processors being connected by point-to-point links as indicated by the edges in the graph. This last observation provides the motivation for the work reported in this paper.

The rest of this paper is organized as follows. Section 2 provides a brief survey of related areas in database processing and graph embeddings. Section 3 provides upper bounds on the cube dimensions for squashed embeddings of completely connected graphs and complete bigraphs. Also, an algorithm for mapping incompletely connected bigraphs is provided. An example of an E-R database schema is given in Section 4, along with a discussion of the advantages and disadvantages of the proposed embedding scheme for parallel database processing. Finally, Section 5 provides conclusions.

\section{SURVEY OF RELATED AREAS}

\subsection{Related Database Work}

Direct encoding of data in the form represented by the E-R schema and the use of a dataflow approach to process these data are both techniques that were used in the Active Graph Model (AGM) machine [6-8]. However, the AGM approach is completely different from the one proposed 

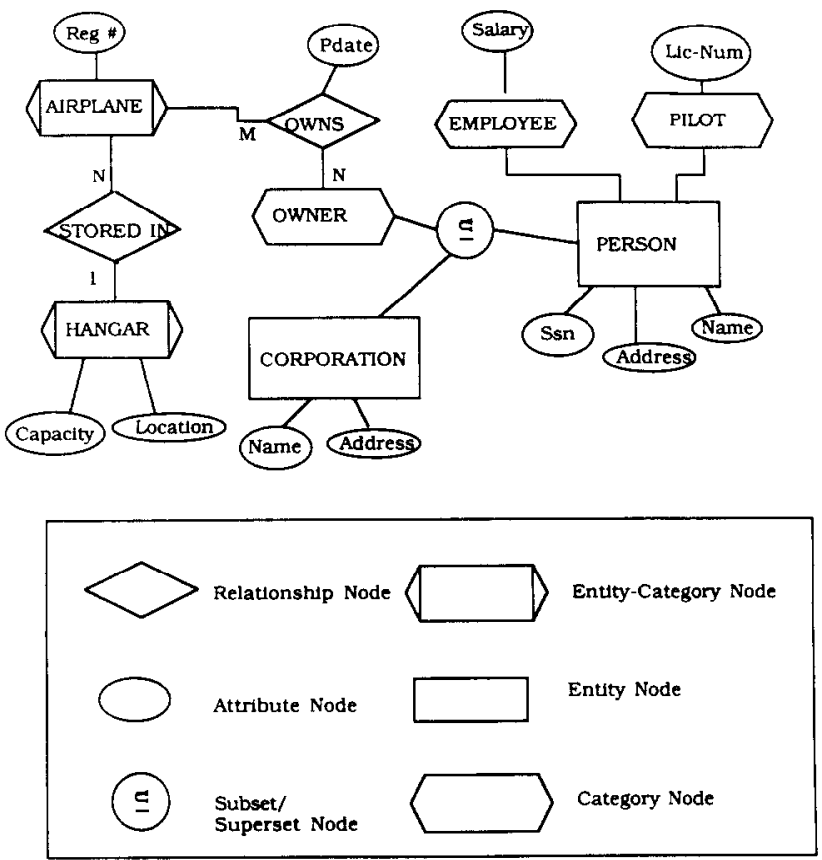

FIG. 1. An ECR logical schema graph for a hypothetical airport database.

here since in AGM each object class (entity or relationship) occurrence is hashed onto a processor. The processors in AGM are connected in the form of a 2-D torus and each processor is a special, dataflow processor. Each occurrence that is selected by the query is routed within the 2-D torus to the location of its related entity (or entities). Each occurrence corresponds to a token in the typical dataflow method of processing. In comparison, the approach proposed here employs macro dataflow processing, where entire sets of selected entity occurrences, rather than individual tokens, are transmitted between nodes. Also, due to the graph mapping technique employed, only the processors containing the data referenced by the query are involved in processing the query, rather than all the processors in the system.

Some issues in relational database processing on hypercube multicomputers have been addressed in our past research [3]. A multicomputer system is one in which each node is an independent computer. As an extension to this research, several parallel algorithms for the relational join operation were implemented on a 64-node NCUBE [4] and parallel query execution strategies were studied via simulations [26]. Further implementations will be carried out on a transputer-based, parallel computer that we have acquired for this project. Another research project that has considered the hypercube interconnection scheme for parallel database processing is [9].

\subsection{Graph Embedding in Hypercubes}

A variety of graph embeddings in hypercubes have been studied. A survey of some of this work is available in [25].
A typical approach is to view the hypercube multicomputer as a processor graph and one can then study the problem of embedding task graphs into this processor graph. In database applications, the query graphs are the task graphs and they are subgraphs of the logical schema graph. The nodes in the query graph represent the objects (entities and/or relationships) and the edges represent the joins (or similar operations) that need to be performed between the objects. In order to keep the communication overhead low, adjacent nodes of the logical schema graph should be assigned to adjacent nodes in $Q_{n}$, if possible.

Several classes of embedding have been proposed to embed a given graph, $G$, into a hypercube, $Q_{d}[20]$. An embedding of a graph $G=(V, E)$ into a $Q_{d}=\left(V^{\prime}, E^{\prime}\right)$ is a one-toone map $\phi$ of $V$ into $V^{\prime}$ such that if $(u, v) \in E$ then $(\phi(u)$, $\phi(v)) \in E^{\prime}$, for all $(u, v) \in E$. A graph is said to be cubical if, for some $d$, there is an embedding of $G$ into $Q_{d}$. If $G$ is cubical, then the least positive number $d$ for which $G$ can be embedded into $Q_{d}$ is called the cubical dimension of $G$. Another class of embeddings is the isomorphic embedding. An algorithm that determines the isomorphism between $G$ and $Q_{d}$ in $O\left(|V| \log _{2}|V|\right)$ steps is proposed in [5]. Isomorphism is a very restrictive embedding class since only graphs that have a hypercube topology are isomorphic to $Q_{d}$.

Cubical embedding requires only that the adjacencies of $G$ be preserved in $Q_{d}$. A graph is said to be cube-critical if it is not cubical but all of its proper subgraphs are cubical [16]. For example, the smallest cube-critical graphs are $K_{3}$ and $K_{2,3}$. A graph is cubical if and only if it contains no cube-critical subgraphs. Other mapping methods have been suggested to embed cube-critical graphs at the expense of dilation cost and expansion cost. Dilation cost is the cost incurred due to adjacent nodes in $G$ not being adjacent to $Q_{d}$. Expansion cost is a measure of the processor utilization in $Q_{d}$ and is the ratio of the number of nodes in $Q_{d}$ to the number of nodes in $G$. These mapping methods include the class of embeddings where nodes in $G$ are mapped to subcubes rather than nodes of $Q_{d}$. A subcube of $Q_{d}$ can be represented by a vector $\left(x_{1}, x_{2}, \ldots, x_{d}\right)$, where $x_{i} \in\{1,0$, $\star\}$, for all $1 \leqslant i \leqslant d$, and $\star$ denotes a coordinate value that is either 1 or 0 . Given two subcubes $X=\left(x_{1}, x_{2}, \ldots, x_{d}\right)$ and $Y=\left(y_{1}, y_{2}, \ldots, y_{d}\right)$ the distance between the two subcubes along the $i$ th dimension, $D_{i}(X, Y)$, is 1 if $\left\{x_{i}, y_{i}\right\}$ $=\{1,0\}$; otherwise it is 0 . Thus, the Hamming distance between $X$ and $Y$ is given by $D(X, Y)=\sum_{i=1}^{d} D_{i}(X, Y)$. The vectors $X$ and $Y$ are said to be adjacent if and only if $D(X, Y)=1$.

A squashed-cube embedding, a concept due to Graham and Pollak [18], is a one-to-one homomorphism from $V$ into a set of mutually disjoint subcubes which preserves the Hamming distance as defined above. The minimum dimension of the hypercube required for a squashed-cube embedding of $G$ is called the weak cubical dimension (WCD), denoted here as SQ-WCD, of $G$ [11]. Winkler [31], showed 
that every connected graph, $G_{n}$, consisting of $n$ nodes has a distance-preserving squashed-cube embedding in a hypercube $Q_{n-1}$. In particular, $K_{n}$ can be squashed-cube embedded into $Q_{n-1}$.

Chen [11] introduced a new class of embedding called relaxed squashed-cube ( $R S)$ embedding which only preserves the adjacencies in $G$. The minimum hypercube dimension required for this embedding will be denoted as RSWCD. The RS embedding problem is similar to the squashed-cube embedding problem and Chen conjectured that it is NP-complete. RS embedding requires hypercubes of considerably lesser dimension than cubical embedding, and every graph can be RS embedded into a hypercube. However, the method in [11] results in a RS-WCD of $(n-1)$ for $K_{n}$, thus requiring an exponential number of processors to embed $K_{n}$.

In studying the problem of state assignment in asynchronous sequential machines, Huffman $[22,23]$ used Karnaugh maps [24] to show that $K_{n}$ can be mapped onto a cube, $Q_{d}$, of dimension $d=2\left\lceil\log _{2} n\right\rceil-1$, such that each node in $K_{n}$ is assigned to two adjacent subcubes. This is a less restrictive form of RS embedding which we call relaxed extended squashed or RES embedding and the cube dimension required in this case is called the RES-WCD. For a complete bipartite graph, $K_{m, n}$, this approach provides an RS-WCD of $\left\lceil\log _{2} m\right\rceil+\left\lceil\log _{2} n\right\rceil$. Thus, the dimension of the cube required for adjacency-preserving mapping is now $O\left(\log _{2} n\right)$ rather than $O(n)$, where $n$ is the number of nodes in the graph. Note that E-R schema graphs are, by definition, bipartite.

\section{EMBEDDING COMPLETELY CONNECTED GRAPHS}

This section discusses the embedding of completely connected graphs and completely connected bigraphs in hypercubes. First, some relevant definitions and terminology are presented.

\subsection{Definitions}

Let $\sigma$ refer to a string of length $d \geqslant 0$, called a $d$-tuple, where each symbol in the string belongs to the set $\{0,1, \star\}$. Thus, $\sigma$ is a member of $\{0,1, \star\}^{d}$ and represents a subcube in $Q_{d}$.

Definition 3.1. Two distinct $d$-tuples, $\sigma_{1}$ and $\sigma_{2}$, are said to be adjacent iff the Hamming distance between them is 1 .

DEFINITION 3.2. Let $\sigma_{1}, \sigma_{2} \in S$, where $S$ is a nonempty set of $d$-tuples. The pair $\left(\sigma_{1}, \sigma_{2}\right)$ is said to be connected if there exists a sequence of adjacent $d$-tuples of $S$ that starts with $\sigma_{1}$ and ends with $\sigma_{2}$. The set $S$ is said to be a connected set if each pair of elements $\sigma_{i}$ and $\sigma_{j} \in S$ is connected.

For example if $S=\{0000 \star, 0010 \star, 0110 \star, 0111 \star\}, \sigma_{1}$ $=0000 \star, \sigma_{2}=0111 \star$, then $\sigma_{1}$ and $\sigma_{2}$ are connected (via $0010 \star$ and $0110 \star$ ) and $S$ is a connected set. Thus, a connected set $S$ containing $|S|$ tuples represents $|S|$ subcubes of $Q_{d}$ such that each subcube is adjacent to at least one other subcube in $S$.

Definition 3.3. Two connected sets $S_{1}$ and $S_{2}$ are said to be adjacent if there exists a $\sigma_{1} \in S_{1}$ and a $\sigma_{2} \in S_{2}$ such that $\sigma_{1}$ and $\sigma_{2}$ are adjacent.

DEFINITION 3.4. Let the connected sets $S_{1}, S_{2}, \ldots, S_{n}$ be assigned to nodes $1,2,3, \ldots, n$, respectively, of a graph, $G_{n}$, of $n$ nodes. This assignment is said to be adjacency preserving if, for any two adjacent vertices $i, j$ of $G_{n}, i \neq j, S_{i}$ is adjacent to $S_{j}$.

An $n$-variable Karnaugh map (or K-map) consists of $2^{n}$ cells representing all possible combinations of these variables [24]. For example, Fig. 2 illustrates a five-variable Kmap. Cyclic code is used to list the combinations as column and row headings. As a result of this coding, cells which have a common side correspond to combinations that differ by the value of just a single variable. Consequently, these cells are adjacent to each other. In general, a cell in an $n$ variable K-map is adjacent to $n$ different cells. The K-map can be folded along a $y$-variable in order to determine the adjacent cell of a cell corresponding to that $y$-variable. For example, in Fig. 2, the cell in the first row, second column is adjacent to the cell in the first row, seventh column along the $y_{1}$-variable that corresponds to the first dimension in a $Q_{5}$.

To embed $K_{n}$ into $Q_{d}$, every pair of nodes, $i$ and $j$, in $K_{n}$ is assigned connected sets, $S_{i}$ and $S_{j}$, in $Q_{d}$ such that $S_{i} \cap S_{j}$ $=\phi$, for all $i \neq j$, and $\left|S_{i}\right|=2,1 \leqslant i, j \leqslant n$, where $n$ is the number of nodes in the graph.

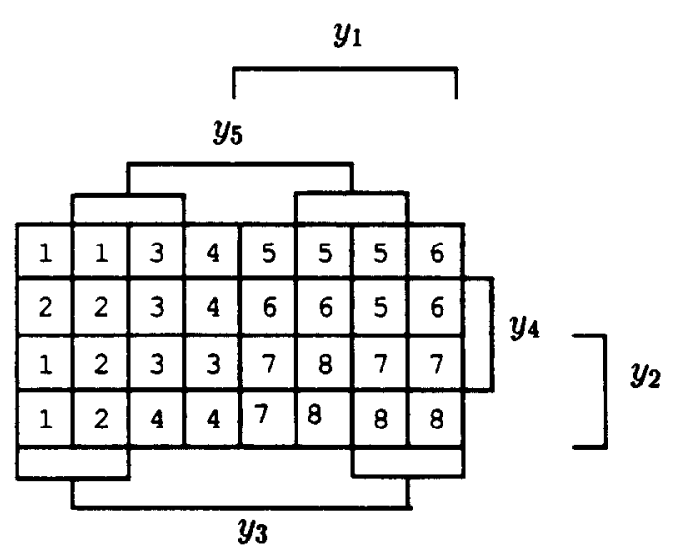

FIG. 2. RES embedding of a $K_{8}$ in $Q_{5}\left(d=2 \log _{2} 8-1\right)$. Nodes in $V$ are labeled 1 through 8 . 


\subsection{Embedding $K_{n}$ into $Q_{d}$}

The RS-WCD of $K_{n}$ is ( $\left.n-1\right)$ using the methods of [11]. Here, we use methods presented in [22, 23] to obtain an RES embedding of $K_{n}$ which gives an RES-WCD of 2 $\times \log _{2} n-1$. This result is obtained by considering a $K_{n}$, where $n=2^{k}$. First, $K_{2}$ can be trivially embedded in $Q_{1}$ ( $d$ $=2 \times 1 \quad 1=1)$. Next, to embed $K_{4}$, at least a cube of dimension 2 is needed. Nodes in $Q_{2}$ have 2-bit addresses and $Q_{2}$ can be represented by a two-variable K-map. Each cell in the K-map is assigned a distinct node. However, it is not possible to embed $K_{4}$ in $Q_{2}$ due to the following property.

Property 3.1. Nodes assigned to opposite corners in a two-variable K-map are not adjacent.

Now, consider a K-map of three variables, $y_{1} y_{2} y_{3}$ (i.e., a $Q_{3}$ ). The columns are identified by odd-subscripted variables and the rows by even-subscripted variables. Each nodc in $K_{4}$ is assigned to two adjacent cells, i.e., a $Q_{1}$. The left half of the K-map can be represented as rows and the right half as columns. Now, any assignment of the four nodes to the two rows and two columns will result in an RS embedding due to the following.

Property 3.2. Each cell in the row on the left-hand side is adjacent to a cell from a distinct column on the righthand side.

Thus, for $K_{4}$, we have found an embedding in which each node is assigned to one subcube. The result of extending this embedding to $K_{8}$ is shown in the five-variable K-map $(d=2 * 3-1)$ of Fig. 2. In general, to embed $K_{2}{ }^{k}$ we need a $Q_{d}$, where $d=2 k-1$ and each node is mapped to a connected set containing $2^{k-1}$ nodes. Thus, the following theorem.

THEOREM 3.1. There exists an RES embedding of $K_{n}$ into $Q_{d}$, where $d=2 k-1$ and $k=\left\lceil\log _{2} n\right\rceil$.

Since the RES-WCD of $K_{n}$ is logarithmic in $n$, large graphs can be embedded on reasonable sized hypercubes as compared to previous results. For example, the RS-WCD for $K_{32}$ is 31 according to [11], thus requiring a hypercube of $2^{31}$ nodes, while the RES-WCD is only 9. Also, the dimensions of the adjacent subcubes assigned by this method are $O\left(\log _{2} n\right)$ rather than $O(n)$. Finally, since the lower bound on any WCD for $K_{n}$ is obviously $\left\lceil\log _{2} n\right\rceil$ and the above theorem now provides an upper bound of $\left(2 \log _{2} n\right)$ -1 , there are $\log _{2} n-1$ choices for the actual dimension.

\subsection{Embedding $K_{m, n}$ into $Q_{d}$}

A method for embedding general graphs in hypercubes is discussed in [11]. The graph is decomposed into simple subgraphs and the RS-WCDs of the simple subgraphs are determined. These subgraphs are then recombined using graph operators to once again obtain the original graph. The RS-WCD of the original graph is obtained as the sum of the RS-WCDs of the simple subgraphs plus an additional term which depends on the graph operators employed. An application of this method to complete bigraphs results in an RSWCD of $\lceil\log m\rceil+\lceil\log n\rceil+1$ for $K_{m, n}$, as shown. Let $m$ $=\left|V_{1}\right|$ and $n=\left|V_{2}\right|$. For each $w_{1} \in V_{1}\left(w_{2} \in V_{2}\right)$, assign a $\left\lceil\log _{2} m\right\rceil\left(\left\lceil\log _{2} n\right\rceil\right)$ bits long address, $D V_{1}\left(w_{1}\right)\left(D V_{2}\left(w_{2}\right)\right)$. Assign each node $w \in G_{m, n}$ a subcube address according to the following rules:

1. If $w_{1} \in V_{1}$, then assign $w_{1}$ a $D$ bits long address $0 \star \star \star \ldots D V_{1}\left(w_{1}\right)$ which contains $\left\lceil\log _{2} n\right\rceil$ consecutive $\star$ 's, and $D V_{1}\left(w_{1}\right)$ is the $\left\lceil\log _{2} m\right\rceil$ bits long address.

2. If $w_{2} \in V_{2}$, then assign $w_{2}$ a $D$ bits long address $1 D V_{2}\left(w_{2}\right) \star \star \star \ldots \star$ which contains $\left\lceil\log _{2} m\right\rceil$ consecutive $\star$ 's and $D V_{2}\left(w_{2}\right)$ is the $\left\lceil\log _{2} n\right\rceil$ bits long address.

If $w_{1}$ is adjacent to $w_{2}$ then there exists a subcube, viz., $0 D V_{2}\left(w_{2}\right) D V_{1}\left(w_{1}\right)$, that is adjacent to the subcube $1 D V_{2}\left(w_{2}\right) D V_{1}\left(w_{1}\right)$ in $w_{2}$. For example, consider embedding $K_{4,4}$, which requires a $Q_{5}(d=2+2+1)$. Nodes in $V_{1}$ are assigned subcubes $0 \star \star 00,0 \star \star 01,0 \star \star 10$, and $0 \star \star 11$ and nodes in $V_{2}$ are assigned subcubes $100 \star \star, 101 \star \star, 110 \star \star$, and $111 \star \star$.

K-map-based methods to embed $K_{m, n}$ are presented below. First, the result for embedding $K_{m, n}$, when $m+n=2^{k}$, is obtained. The generalization to any $K_{m, n}$ follows. Note that the following methods provide RS embeddings and not RES embeddings, as in the case of $K_{n}$. Lemmas 3.1 and 3.2 consider two different cases for $K_{m, n}$.

LEMMA 3.1. $A K_{m, n}$ can be RS embedded into $Q_{d}$, where $m=n=2^{k-1}, d=2 k-2$, and $k>1$.

Proof. The proof is by construction. Embedding $K_{m, n}$ when $n=m=1$, is trivial. For $m>1$, let $K_{m, n}=\left(\left(V_{1}, V_{2}\right)\right.$, $E),\left|V_{1}\right|=\left|V_{2}\right|=2^{k-1}$, and $\left\lceil\log _{2} m\right\rceil+\left\lceil\log _{2} n\right\rceil=2(k-1)$. Construct a $(2 k-2)$-variable K-map. Let the $y_{1} y_{2}$-variables designate quadrants of the K-map. Assign one row of quadrant $y_{1} y_{2}=00$ to each of the first $2^{k-2}$ nodes of $V_{1}$ and assign the remaining $2^{k-2}$ nodes of $V_{1}$ to quadrant $y_{1} y_{2}=11$ in a similar fashion. Clearly, these nodes have been assigned to subcubes which are adjacent to every column (subcube) in quadrants 01 and 10 (Property 3.2). Each column in quadrants 10 and 01 is assigned to a distinct node of $V_{2}$. The adjacency requirements of $K_{m, n}$ are satisfied by this assignment. Each quadrant contains $m / 2=2^{k-2}$ nodes, thus requiring $(k-2) y$-variables. Two quadrants each are required for $V_{1}$ and $V_{2}$, thus requiring $2(k-1)$ or $2 k \cdot 2 y$ variables for all the nodes in $V_{1}$ and $V_{2}$.

For example, Fig. 3 shows the embedding of $K_{8,8}$ in $Q_{6}(d$ $=2 \times 4-2$ ). Nodes in the node sets, $V_{1}$ and $V_{2}$, are labeled from 1 to 8 and $a$ to $h$, respectively. 
$\geqslant m$. Thus, $\left\lceil\log _{2}(m+n)\right\rceil \geqslant j_{2}$. According to Theorem 3.2, the RS-WCD of $K_{m, n}$ is $j_{1}+j_{2}$ and since $G_{m, n}$ is a subgraph of the $K_{m, n}$, the RS-WCD of $G_{m, n}$ is $\leqslant j_{1}+j_{2}$. Furthermore, since any $G_{m, n}$ requires at least a hypercube of dimension $\left\lceil\log _{2}(m+n)\right\rceil$, we have $j_{2} \leqslant$ RS-WCD of $G_{m, n} \leqslant j_{1}+j_{2}$.

Consider a graph, $G_{2^{j_{1}, 2_{2}}}=\left(\left(V_{1}, V_{2}\right), E\right)$, which has the same edge set as $G_{m, n}$. The algorithm for RS embedding $G_{2^{j_{1}}, 2_{2}}$ is presented. Let $C_{i}, i \in V_{1}$, denote the adjacency set of a node $i$; i.e. $C_{i}=\left\{k \mid k \in V_{2}\right.$ and there is an edge between $i$ and $k\}$. Similarly, let $C_{j}^{\prime}, j \in V_{2}$, denote the adjacency set of node $j$.

Let $V_{1}^{1}, V_{1}^{2}, \ldots, V_{1}^{2_{1}^{j_{1}-i+1}}\left(V_{2}^{1}, V_{2}^{2}, \ldots, V_{2}^{2 j_{-}-i+1}\right)$ denote $2^{j_{1}-i+1}\left(2^{j_{2}-i+1}\right)$ partitions of the node set $V_{1}\left(V_{2}\right)$, for $1 \leqslant i$ $\leqslant j_{1}$, such that for each partition $V_{1}^{k}$ of $V_{1}$ the following is true. For every $u, v \in V_{1}^{k}$ and $s \ni V_{1}^{k}$ :

$$
\left|C_{u} \cap C_{v}\right| \geqslant\left|C_{u} \cap C_{s}\right|
$$

and

$$
\left|V_{1}^{k}\right|=2^{i-1}
$$

Each partition, $V_{2}^{k}$, of $V_{2}$ is obtained by similar conditions. For each partition $V_{1}^{t}, 1 \leqslant t \leqslant 2^{j_{1}-i+1}$, let $U_{t}$ denote the set

$$
U_{t}=\left\{V_{2}^{j} \mid\left(\bigcup_{s \in V_{1}^{t}} C_{s}\right) \cap V_{2}^{j} \neq \phi, 1 \leqslant j \leqslant 2^{j_{2}-i+1}\right\} .
$$

Two partitions $V_{1}^{t}$ and $V_{2}^{j}$ are connected if there is an

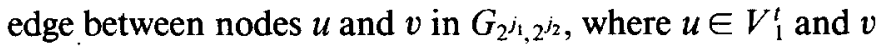

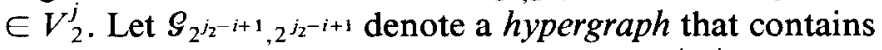
one node each for every partition of $V_{2}$ and $2^{j_{2}-j_{1}}$ nodes for every partition of $V_{1}$. Let $V_{1}^{t k}, 1 \leqslant k \leqslant 2^{j_{2}-j_{1}}$, denote the $2^{j_{2}-j_{1}}$ nodes representing each partition $V_{1}^{t}$. Connect $V_{1}^{t k}$ nodes of each partition $V_{1}^{t}$ in a hypercube topology of dimension $j_{2}-j_{1}$ in the hypergraph $g_{2^{j_{2}-i+1}, 2_{2} j^{-i+1}}$. Connect each $V_{1}^{t k}$ to $\left|U_{t}\right| / 2^{j_{2}-j_{1}}$ distinct $V_{2}^{j} \in U_{t}$ nodes. Based on the topology of the hypergraph, the next lemma states sufficient conditions for embedding a $G_{2^{j_{1}, 2_{2}}}$ into a $Q_{d}$, where $j_{2}+1 \leqslant d \leqslant j_{1}+j_{2}$.

LEMMA 3.3. If the hypergraph $S_{2^{j_{2}-i+1}, 2^{j_{2}-i+1}}$ is isomorphic to $Q_{j^{-i+2}}$ then $G_{2^{j_{1}}, 2^{j_{2}}}$ can be $R S$ embedded on a hypercube $Q_{j_{2}+i}$.

Proof. Since $\mathscr{S}_{2^{j_{2}-i+1}, 2^{j_{2}-i+1}}$ is isomorphic to $Q_{j^{-i+2}}$, there exists an embedding of $g_{2^{j_{2}-i+1}, 2^{j_{2}-i+1}}$ such that all the adjacencies in it are satisfied. Note that the graph $g_{2^{j_{2}-i+1}, 2^{j_{2}-i+1}}$ contains $2^{j_{2}-j_{1}}$ nodes for every partition of $V_{1}$ such that if two partitions $V_{1}^{t}$ and $V_{2}^{j}$ are connected then there is a node corresponding to $V_{1}^{t}$, namely $V_{1}^{t k}$ in $\boldsymbol{g}_{2^{j_{2}-i+1}, 2^{j_{2}-i+1}}$, that is connected to $V_{2}^{j}$. Therefore, all the adjacencies in the partition $V_{1}^{t}$ are satisfied. Now, let $K_{1}$ be the K-map used to embed $S_{2^{j_{2}-i+1}, 2^{j_{2}-i+1}}$ into $Q_{j_{2}-i+2}$. Assign a $j_{2}$ $-j_{1}$ dimension subcube each in $K_{1}$ to every partition $V_{1}^{t}$ of $V_{1}$. Assign one node each to every partition $V_{2}^{j}$ of $V_{2}$. Each cell of $K_{1}$ is a $\mathrm{K}$-map of $(2 i-2) y$-variables. Assign one row (column) of a cell to each node in $V_{1}^{t k}\left(V_{2}^{j}\right)$.

The requirement that the hypergraph be isomorphic to a hypercube is, in fact, too restrictive. In general, it is sufficient that the graph $S_{2^{j_{2}-i+1}, 2^{j_{2}-i+1}}$ be embeddable in $Q_{j_{2}-i+2}$, i.e., that the hypergraph is cubical. However, the problem of deciding whether the graph $G_{2^{d}}$ is embeddable in $Q_{d}$ is NP-complete [25]. While it has been reported that Krumme [25] has found an embedding for such graphs into $Q_{d+1}$, the authors are not aware of the characterization of such graphs. If such an embedding is available, it can be used to determine whether the hypergraph is embeddable in $Q_{j_{2}-i+3}$. The following algorithm finds an RS embedding for $G_{2^{j_{1}, 2}} j_{2}$ using the above lemma.

\section{Algorithm Incomplete}

(* Embedding an incomplete $G_{2^{j_{1}}, 2_{2}}$ in a hypercube *)

1. Assign $G=G_{2^{j_{1}}, 2^{j_{2}}}=\left(\left(V_{1}, V_{2}\right), E\right)$; and $S=\phi$.

$\left(* g\right.$ denotes the hypergraph of Lemma $3.3^{*}$ )

2. For $i=1,2, \ldots, j_{1}$ do:

(a) Construct the set $C_{t}$ for every $t \in V_{1}, V_{2}$.

(b) Generate $2^{j_{1}-i+1}\left(2^{j_{2}-i+1}\right)$ partitions of $V_{1}\left(V_{2}\right)$ according to (1) and (2).

(c) Generate $U_{t}$ for every $t \in V_{1}$.

(d) Construct the hypergraph $\mathscr{S}_{2^{j_{2}}-i+1} 2^{j_{2}-i+1}$.

(e) If $S_{2^{j_{2}-i+1}, 2^{j} 2^{-i+1}}$ is isomorphic to $Q_{j_{2}-i+2}$ then an RS embedding has been found for $G_{2^{j_{1}}, 2^{j_{2}}}$ on $Q_{j_{2}+i}$ else $G=\mathscr{S}_{2^{j_{2}-i+1}, 2^{j_{2}-i+1}} ; \boldsymbol{S}_{2^{j_{2}-i+1}, 2^{j_{2}-i+1}}=\phi$.

Step 2(b) takes $O\left(n^{3}\right)$ time, where $n$ is the number of nodes in $G$. The graph size reduces by a factor of 2 in each iteration. In the worst case, there are $j_{1}$ iterations; i.e., the running time is $O\left(n^{3} j_{1}\right)$, where $n=2^{j_{1}}+2^{j_{2}}$ and $j_{2} \geqslant j_{1}$.

For example, a $G_{16,16}$ is represented in Fig. 5. The algorithm attempts to embed the graph in $Q_{5}\left(d=j_{2}+1=5\right)$ and determines that it cannot be embedded. Next, the node sets $V_{1}$ and $V_{2}$ are each partitioned into eight partitions, $\{\{a, b\},\{c, d\},\{e, f\},\{g, h\},\{i, j\},\{k, l\},\{m, n\}$, $\{o, p\}\}$ and $\{\{1,2\},\{3,4\},\{5,6\},\{7,8\},\{9,10\},\{11$, $12\},\{13,14\},\{15,16\}\}$, respectively. The corresponding $g_{8,8}$ is generated and is represented in Fig. 6. The $g_{8,8}$ is isomorphic to $Q_{j_{2}-i+2}=Q_{4-2+2}=Q_{4}$. Thus, the RS-WCD of the required hypercube is $j_{2}+i=4+2=6$ (see Fig. 7).

\section{DATABASE PROCESSING ISSUES}

\subsection{Mapping E-R Schema Graphs}

The Entity-Relationship model views the real world as consisting of entities and relationships. An entity is an object which can be distinctly identified and is described by a set of attributes. Entities are grouped into entity sets, $E_{i}$ 's, 


$$
\begin{aligned}
& \{a: 1,2,3,4,7,8,9,10\} ;\{b: 1,2,3,4,7,8,9,10\} \\
& \{c: 1,2,3,4,5,6,11,12\} ;\{d: 1,2,3,4,5,6,11,12\} \\
& \{e: 3,4,5,6,7,8,15,16\} ;\{f: 3,4,5,6,7,8,15,16\} \\
& \{g: 1,2,5,6,7,8,13,14\} ;\{h: 1,2,5,6,7,8,13,14\} \\
& \{i: 1,2,9,10,11,12,13,14\} ;\{j: 1,2,9,10,11,12,13,14\} \\
& \{k: 3,4,9,10,11,12,15,16\} ;\{l: 3,4,9,10,11,12,15,16\} \\
& \{m: 5,6,11,12,13,14,15,16\} ;\{n: 5,6,11,12,13,14,15,16\} \\
& \{o: 7,8,9,10,13,14,15,16\} ;\{p: 7,8,9,10,13,14,15,16\}
\end{aligned}
$$

FIG. 5. An incomplete bipartite graph, $G_{16,16}$. Nodes in $V_{1}\left(V_{2}\right)$ are assigned labels $a$ through $p$ ( 1 through 16). The edges in $E$ are represented by the sets; e.g., $\{a: 1,2,3,4,7,8,9,10\}$ indicates that $a \in V_{1}$ is connected to nodes $1,2,3,4,7,8,9,10 \in V_{2}$.

such that an entity has to satisfy a predicate in order to be a member of an entity set. A relationship is a mathematical relation among $n$ entities such that each entity is taken from an entity set. A set, $R_{i}$, of relationships over a given set of entities is called a relationship set, where $R_{i}=\left\{\left(e_{1}, e_{2}, \ldots\right.\right.$, $\left.\left.e_{n}\right) \mid e_{1} \in E_{1}, \ldots, e_{i} \in E_{i}, \ldots, e_{n} \in E_{n}\right\}$.

An E-R schema graph can be mapped into a hypercube by first converting it into a storage graph, $G$, which is an undirected graph containing a node corresponding to each entity and relationship set in the E-R schema. From the definitions, it is clear that entity sets cannot be contained in, or be constituent classes of, other entity sets. Similarly, relationship sets cannot contain other relationship sets. Thus, by definition, the E-R schema graph and its storage graph, $G$, are bipartite.

Figure 8 shows an E-R schema graph containing the entity sets, Department, Employee, Project, and Supplier, and the relationship sets. Dept-Emp, Proj-Work, Proj-Manager, Supp-Proj, and Proj-Auditor. The meanings of the relationship sets are self-explanatory. For example, ProjAuditor defines the Project-Auditor relationship between the entities Employee and Project. More than one relationship can be defined on given entity sets; e.g., the relationship sets Proj-Work, Proj-Auditor, and Proj-Manager are all

$$
\begin{aligned}
& \{\{a, b\}:\{1,2\},\{3,4\},\{7,8\},\{9,10\}\} \\
& \{\{c, d\}:\{1,2\},\{3,4\},\{5,6\},\{11,12\}\} \\
& \{\{e, f\}:\{3,4\},\{5,6\},\{7,8\},\{15,16\}\} \\
& \{\{g, h\}:\{1,2\},\{5,6\},\{7,8\},\{13,14\}\} \\
& \{\{i, j\}:\{1,2\},\{9,10\},\{11,12\},\{13,14\}\} \\
& \{\{k, l\}:\{3,4\},\{9,10\},\{11,12\},\{15,16\}\} \\
& \{\{m, n\}:\{5,6\},\{11,12\},\{13,14\},\{15,16\}\} \\
& \{\{o, p\}:\{7,8\},\{9,10\},\{13,14\},\{15,16\}\}
\end{aligned}
$$

FIG. 6. The hypergraph, $g_{8,8}$, of the graph in Fig. 5 for the second iteration of the algorithm Incomplete in Section 3.4. $\{x, y\}$ represents a partition of the nodes in $V_{1}$ or $V_{2}$. The edges of $g_{8,8}$ are represented by sets; e.g., $\{\{a, b\}:\{1,2\},\{3,4\},\{7,8\},\{9,10\}\}$ indicates that the partition $\{a, b\}$ is connected to the partitions $\{1,2\},\{3,4\},\{7,8\},\{9,10\}$.

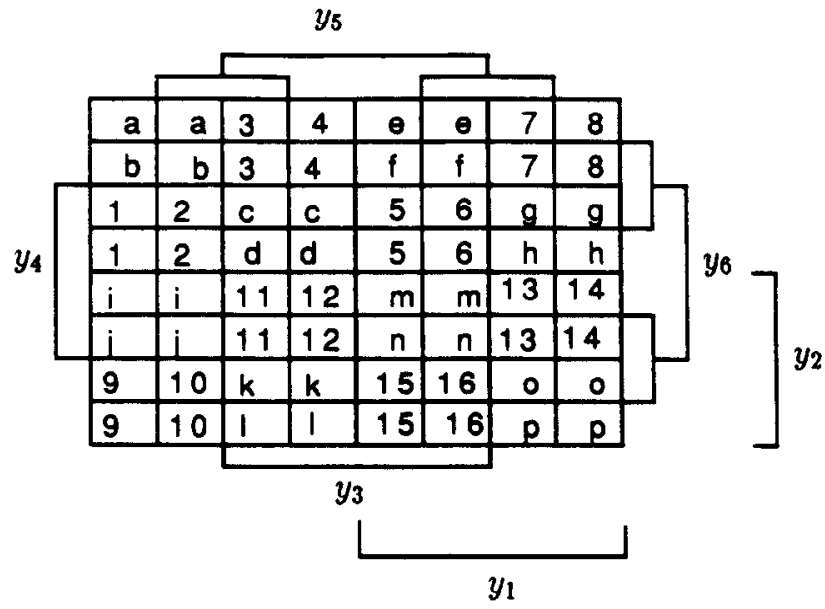

FIG. 7. An RS embedding of the graph of Fig. 5.

defined on the entity sets Project and Employee. The cardinality of the various relationships between entity sets can be $1: 1,1: n$, or $m: n$. For example, Proj-Auditor is a $1: n$ relationship between Employee and Project. This implies that a single employee can be the auditor for more than one project and each project has only a single auditor. While the cardinality of relationships and other semantics do not effect embedding, they need to be accounted for by the data storage and processing strategies. Figure 9 shows the corresponding storage graph for the schema. The graph for this example is a $G_{4,5}$ that contains a cube-critical graph, $K_{2,3}$. The lower bound on the cube dimension for this graph is 4 . The embedding algorithm starts with $G_{4,8}$ and embeds it in $Q_{5}$, as shown in Fig. 10. This example illustrates several fea-

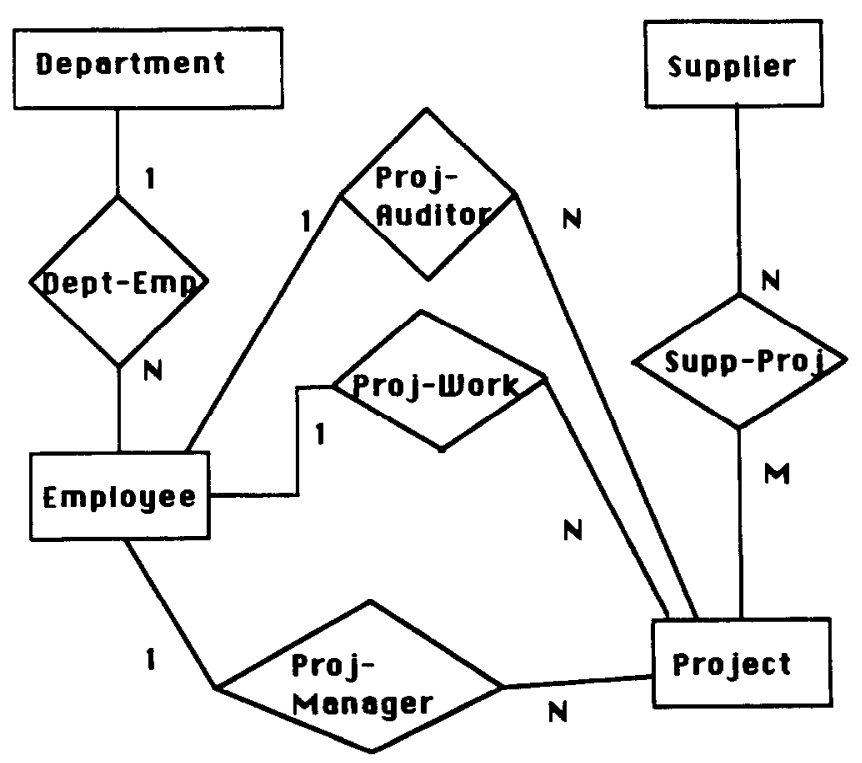

FIG. 8. A sample E-R logical schema graph. 
tures of the RS embedding for E-R schemas which are discussed in the next section.

\subsection{Ramifications of RS Embedding}

We began sludying the problem of embedding logical schema graphs into processor graphs in order to solve the data placement problem for parallel database systems. The intent was to find an embedding scheme that minimizes communication costs in a multicomputer system. Squashed embedding is preferred because (1) it distributes data across subcubes, thereby providing parallel I/O capability. (2) Queries can be represented as subgraphs of the schema graph. Only the dataflow directions in the query graph need to be determined at run time, based on the selection predicates in the query. We are currently studying various aspects of this problem. (3) It should be possible to include the dataflow concurrency control methods that have been proposed in the literature [14] into this dataflow processing scheme. (4) The reliability of the database system could be enhanced in several ways. Failure of a node and/ or link implies that the corresponding entity or relationship node is no longer accessible in the logical schema graph. Thus, future queries could be classified as being fully answerable, partially answerable, or unanswerable. (5) Once a schema graph is mapped, one or more replicas of the entire database can be easily mapped onto the same hypercube by "skewing" each copy, by applying a simple address transformation at each node. Thus, each node in the hypercube knows the exact location of its copies.

However, direct embedding of logical schema graphs into processor graphs, using adjacency-preserving techniques, has several other ramifications for database processing. First, there is little control over the size of the subcube assigned to each node in the schema graph. Thus, it is not possible to choose subcube sizes in proportion to, say, the amount of data represented by each node. Second, the embedding may result in unused subcubes, as in Fig. 10. In general, squashed embedding may have to be used along with other schemes to obtain a good mapping. The above issues are all topics for future research.

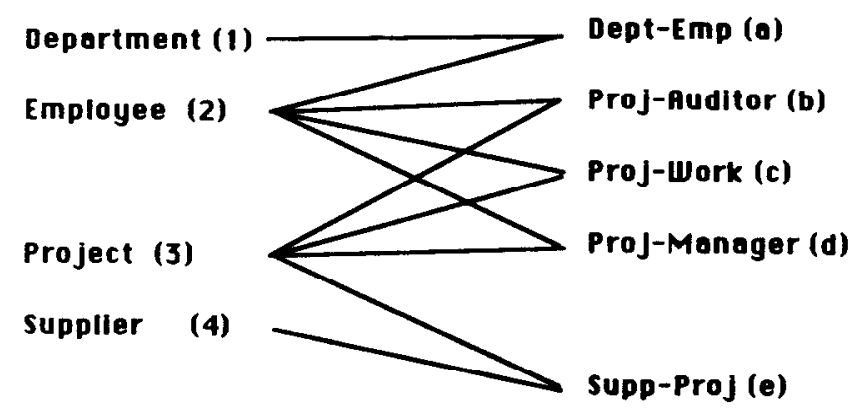

FIG. 9. The $G_{4,5}$ storage graph corresponding to the E-R schema graph of Fig. 8.

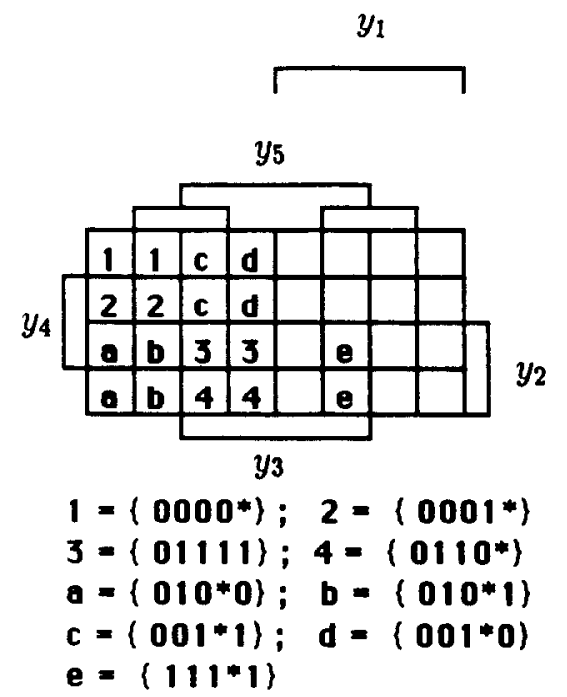

FIG. 10. An RS embedding of the $G_{4.5}$ of Fig. 9 into a $Q_{5}$.

\section{CONCLUSION}

A class of adjacency-preserving, squashed embeddings was studied with the idea of using such embeddings to directly map E-R schema graphs onto hypercubes. Results for relaxed extended squashed embedding of complete graphs and relaxed squashed embedding of complete bigraphs were obtained using a technique that was used in the state assignment of asynchronous sequential machines by Huffman. The minimum cube dimension required for such embedding is called the weak cubical dimension of the graph. For $K_{n}$, an RES-WCD of $O\left(\log _{2} n\right)$ is obtained. For $K_{m, n}$, the RS-WCD is $\left\lceil\log _{2} m\right\rceil+\left\lceil\log _{2} n\right\rceil$. Thus, the bounds on the RS-WCD of $G_{m, n}$ are $d_{\min }=\left\lceil\log _{2}(m+n)\right\rceil \leqslant$ RSWCD $\leqslant\left\lceil\log _{2} m\right\rceil+\left\lceil\log _{2} n\right\rceil=d_{\max }$. An algorithm for determining the RS-WCD of incomplete bipartite graphs was presented which examines the $d_{\max }-d_{\min }$ choices to be examined to obtain the best cube dimension. However, while preserving adjacency is important when mapping schema graphs, another important factor is the loading of each node/subcube. In general, algorithms for embedding should consider this factor also. Overall, this embedding approach provides several opportunities for novel query processing, concurrency control, and reliability methods in parallel database systems.

\section{REFERENCES}

1. Abiteboul, S., and Hull, R. IFO: A formal semantic database model. ACM Trans. Database Systems 12, 4 (Dec. 1987), 525-565.

2. Banerjee, J., Hsaio, D. K., and Kannan, K. DBC: A database computer for very large databases. IEEE Trans. Comput. C-28, 6 (June 1979).

3. Baru, C. K., and Frieder, O. Database operations in a cube-connected multicomputer system. IEEE Trans. Comput. C-38, 6 (June 1989), 920-927. 
4. Baru, C. K., Padmanahhan, S., Stout, Q., and Wagar, B. A comparison of join and projection algorithms for hypercubes. Proc. 4th Conference on Hypercube Concurrent Computers and Applications, Monterey, CA, Mar. 6-8, 1989.

5. Bhat, K. V. S. On complexity of testing a graph for n-cube. Inform. Process. Lett. 11, 1 (Aug. 1980), 16-19.

6. Bic, L., and Hartmann, R. L. Simulated performance of a data-driven database machine. J. Parallel Dist. Comput. 3 (1986), 1-22.

7. Bic, L., and Rasset, T. L. Performance of a relational dataflow machine. Proc. Hawaii International Conference on System Sciences, Jan. 1986, pp. 1-22.

8. Bic, L., and Hartmann, R. L. AGM: A dataflow machine. Tech. Rep. Department of Information and Computer Science, University of California, Irvine, Feb. 1987.

9. Bratbergsengen, K. Algebra operations on a parallel computer: Performance evaluation. Proc. 5th International Workshop on Database Machines, Karuizawa, Japan, Oct. 1987.

10. Chen, P. P. S. The entity-relationship model: Toward a unified view of data. ACM Trans. Database Systems 1, 1 (Mar. 1976), 9-36.

11. Chen, M. S., and Shin, K. G. Embedding cooperative task modules into a hypercube multiprocessor. Proc. 2nd Conference on Hyp. Conc. Computers and Applications, Monterey, CA, 1987, pp. 122-129.

12. DeWitt, D. J. DIRECT: A multiprocessor organization for supporting relational Database Management Systems. IEEE Trans. Comput. C28, 6 (June 1979), 395-408.

13. DeWitt, D. J., et al. GAMMA: A high performance dataflow database machine. Proc. I2th International Conference on Very Large Data Bases, Kyoto, Japan, Aug. 1986, pp. 228-237.

14. Eich, M. H., and Wells, D. L. Database concurrency control using data flow graphs. ACM Trans. Database Systems 13, 2 (June 1988), 197227.

15. Elmasri, R., Weeldreyer, J., and Hevner, A. The category concept: An extension to the entity-relationship model. Data and Knowledge Engineering, Elsevier/North-Holland, New York, 1985, Vol. 1.

16. Garey, M. R., and Graham, R. L. On cubical graphs. J. Combin. Theory Ser. B 18 (1975), 84-95.

17. Graham, R. L., and Pollak, H. O. On the addressing problem for loop switching. Bell System Tech. J. 50, 8 (Oct. 1971), 2495-2519.

18. Graham, R. L., and Pollak, H. O. On embedding graphs in squashed cubes. Graph Theory and Application, Lecture Notes in Mathematics Vol. 303. Springer-Verlag, New York/Berlin (Proc. of a conference held at Western Michigan University, May 10-13, 1972).

19. Hammer, M. M., and McLeod, D. J. Database description with SDM: A semantic database model. ACM Trans. Database Systems 6, 3(Sept. 1981).

20. Harary, F., Hayes, J. P., and Wu, H. J. A survey of the theory of hypercube graphs. Comput. Math. Appl. 15, 4 (1988), 277-289.
21. Hillyer, R., and Shaw, D. E. NON-VON's performance on certain database benchmarks. IEEE Trans. Software Engrg. SE-12, 4 (Apr. 1986), 577-583.

22. Huffman, D. A. The synthesis of sequential switching circuits: Part I. J. Franklin Inst. 257 (Mar. 1954), 161-190.

23. Huffman, D. A. The synthesis of sequential switching circuits: Part II. J. Franklin Inst. 257 (Apr. 1954), 275-303.

24. Kohavi, Z. Switching and Finite Automata. McGraw-Hill, New York, 1978.

25. Livingston, M., and Stout, Q. F. Embeddings in hypercubes. Math Comput. Modelling 11 (1988), 222-227.

26. Padmanabhan, S., and Baru, C. K. Single query execution in multicomputer database systems. Tech. Rep., Adv. Comp. Arch. Lab., University of Michigan, Ann Arbor, MI.

27. Su, S. Y. W. SAM*: A semantic association model for corporate and scientific-statistical databases. Inform. Sci. 29 (1983), 151-199.

28. Su, S. Y. W. Database Computers. McGraw-Hill, New York, 1988.

29. Teorcy, T. J., Yang, D., and Fry, J. P. A logical design methodology for relational databases using the extended entity-relationship model. ACM Comput. Surveys 18, 2 (June 1986), 197-222.

30. Verheijen, G. M. A. NIAM-An information analysis method. Information Systems Department, Control Data B V, The Netherlands, 1976.

31. Winkler, P. M. Proof of the squashed cube conjecture. Combinatorica 3, 1 (1983), 135-139.

CHAITANYA K. BARU received the bachelor's degree ( B.Tech.) from the Indian Institute of Technology, Madras, India, in 1979 and the master's (M.E.) and Ph.D. degrees from the University of Florida, Gainesville, Florida, in 1983 and 1985, all in electrical engineering. He has been an assistant professor in the Department of Electrical Engineering and Computer Science at the University of Michigan, Ann Arbor, Michigan, since 1985. His research interests are in the areas of database systems, parallel processing, and database machines. One of his current projects is the implementation of an experimental database system on a transputer-based parallel computer. His research is currently funded by the National Science Foundation.

PIYUSH GOEL received a bachelor's degree in electronics and communication engineering from the University of Roorkec, Roorkcc, India, in 1982 and a master's degree in electrical and computer engineering from Wayne State University, Detroit, Michigan, in 1984 . He is currently pursuing a Ph.D. degree in the Department of Electrical Engineering and Computer Science, University of Michigan, Ann Arbor, Michigan. His current research is related to parallel processing of databases based on semantic data models and query execution strategies and concurrency control issues in such systems. 